\title{
A Dorsal Approach for Ulnar Shortening Osteotomy
}

\author{
Bo J. W. Notermans, $\mathrm{MS}^{1} \quad$ Wiesje Maarse, MD, $\mathrm{PhD}^{1} \quad$ Arnold H. Schuurman, MD, $\mathrm{PhD}^{1}$ \\ ${ }^{1}$ Department of Plastic and Reconstructive Surgery, University \\ Medical Center Utrecht, The Netherlands \\ Address for correspondence Arnold H. Schuurman, MD, PhD, \\ Department of Plastic and Reconstructive Surgery, University Medical \\ Center Utrecht Heidelberglaan 100, 3584 CX Utrecht, The \\ J Wrist Surg 2018;7:281-287. \\ Netherlands (e-mail: a.schuurman@umcutrecht.nl).
}

\begin{abstract}
Keywords

- ulnar shortening osteotomy

- dorsal approach

- hardware removal

- ulnar impaction syndrome

Aim This study aims to evaluate the long-term complications, results, and patient satisfaction rates of a dorsally approached ulnar shortening osteotomy for ulnar impaction syndrome.

Methods A retrospective chart review of 20 patients was performed. Primary outcomes of interest were subjective, measured using the Patient-Rated Wrist Evaluation (PRWE) score, Disability of the Arm, Shoulder, and Hand (DASH) score, and the third questionnaire about patient satisfaction, composed by the authors. Secondary outcomes included hardware removal due to irritation and other complications.

Results Mean postoperative functional score of PRWE was 28 (standard deviation [SD], 30) and DASH 20 (SD, 26), respectively. Fifteen patients were satisfied with the operation. Removal of hardware was noted in six patients. In one patient plate breakage occurred.

Conclusion Similar postoperative functional scores and complications were seen in patients undergoing an ulnar shortening osteotomy with a dorsally placed plate for ulnar impaction syndrome, compared with other plate placement localizations. The incidence of plate removal is also comparable to previously described results. As the dorsally placed plate and freehand technique, are relatively easy, we feel that it has a place in the treatment of ulnar impaction syndrome.

Level of Evidence Level IV, retrospective cohort study.
\end{abstract}

In case of excessive ulnar head compression against the ulnar carpal bones and the triangular fibrocartilage complex (TFCC), or as a result of other structural or functional ulnar overlength, an ulnar impaction syndrome may occur. ${ }^{1-3}$ This syndrome is usually associated with positive ulnar variance, ulnocarpal chondromalacia, and degenerative changes of the TFCC, lunate, triquetrum or the ulnar head. Positive ulnar variance can either be congenital, a result of premature physeal arrest or malunited distal radius fractures. ${ }^{1,3,4}$ These problems can cause ulnar-sided wrist pain, less grip strength, and reduced range of motion. ${ }^{5-7}$

Ulnar impaction syndrome can be treated conservatively, but when symptoms persist, surgery might be needed.

received

December 14, 2016

accepted after revision

September 29, 2017

published online

November 22, 2017
Length discrepancies between the ulna and radius may lead to incorrect interaction and painful transmission along the distal part of the upper limb. Changing the length of the ulna, ulnar shortening osteotomy (USO), by only $2.5 \mathrm{~mm}$ will change the load transmission across the wrist to the distal forearm bones dramatically. ${ }^{1,8}$ USO using a volarly or ulnarly positioned plate is the most commonly applied technique. ${ }^{6}$ Unfortunately, the reported incidence of plate removal due to irritation is up to $55 \%$ when using these techniques. ${ }^{5}$ This is possibly caused by greater direct pressure being exerted on the hardware, as the functional position of the forearm is in pronation. Consequently, patients are less satisfied due to soft tissue irritation, which more commonly occurs in volarly
Copyright $\odot 2018$ by Thieme Medical Publishers, Inc., 333 Seventh Avenue, New York, NY 10001, USA. Tel: +1(212) 584-4662.
DOI https://doi.org/ 10.1055/s-0037-1608636. ISSN 2163-3916. 
and ulnarly placed implants. ${ }^{6}$ USO can also be performed using a dorsal approach with placement of the plate on the dorsal surface of the ulna. Placement of plates on the dorsal surface can be performed using a relatively easy freehand technique. This approach may lead to less hardware irritation while being comparable to the volar and ulnar approach with regard to the union, wrist motion, and functional scores. ${ }^{6}$ To evaluate the long-term complications and results of the dorsal approach we retrospectively assessed complication rates and patient satisfaction using the dorsal approach. Furthermore, we studied patients' subjective functional disability and pain after dorsally approached USO, using the Disabilities of the Arm, Shoulder, and Hand (DASH), the Patient-Rated Wrist Evaluation (PRWE), and a patient satisfaction questionnaire. ${ }^{9-11}$

\section{Methods}

\section{Patients}

From 2005 to 2014 a single surgeon performed 49 USO with a dorsal approach. Patients' follow-up had to be at least 12 months for inclusions, as bony union usually takes at least three months. As a result of this, six patients could not be included in this study. Because an accompanying surgery may affect postoperative functional scores, patients were excluded if they underwent a USO with an accompanying surgery. Nine patients also underwent an Adams procedure $(n=5)$, radial styloid resection $(n=1)$, bone grafting $(n=2)$ or a radial osteotomy $(n=1)$. A total of 34 patients were enrolled in this study. At follow-up, patients' age ranges from 18 to 75.

After approval by the medical ethical review committee (reference number: WAG/mb/15/036704), a retrospective chart review was conducted. The primary outcomes of interest were the PRWE score, DASH score, and patients' satisfaction scores. Secondary outcomes included plate removal due to hardware irritation and complications, such as infection, pseudoarthrosis or plate breakage and revision surgery. Medical charts were reviewed to ascertain the occurrence of complications, the amount of ulnar shortening and to determine whether union occurred or an operation for nonunion was necessary. Union was assessed on anteriorposterior and lateral plain radiographs and was defined as blurring of osteotomy margins and periosteal reaction that completely bridges the cortical interruption. However, during early stages of bony union, an osteotomy line can remain visible. $^{12,13}$ Therefore, we used clinical criteria, such as painless range of motion and absence of tenderness at the osteotomy site in combination with radiographic criteria to define union.

\section{Questionnaires}

We used two validated questionnaires; the DASH Dutch Language Version (DASH-DLV) and the PRWE score, to assess functional disability and pain. ${ }^{9}$ The third questionnaire (-Appendix), created by the authors, displays whether or not patients are satisfied with the operation and the final result of the operation. Furthermore, the questionnaire evaluates whether patients would undergo the same surgery again and recommend this surgery. The questions are rated on a five-point scale, in which a low score indicates satisfaction and a high score indicates dissatisfaction.

\section{Surgical Indications}

Patients with ulnar impaction syndrome, as a result of congenital or acquired disorders, such as malunited distal radial fractures, unresponsive to conservative therapy, were indicated for surgical treatment. The diagnosis was confirmed if a positive ulnar variance was present on a plain radiograph. To determine ulnar variance on radiographs, the accepted standard view is a posteroanterior view obtained with the wrist in neutral forearm rotation, the elbow flexed 90 degrees, and the shoulder abducted 90 degrees. It refers to the relative lengths of the distal articular surfaces of the radius and ulna. If positive, for example, the ulna projects more distally than the radius. In this study, the concentric circle method, described in 1982 by Epner et al, was used.

\section{Dorsal Plate Technique}

After axillary nerve blockage or general anesthesia, a tourniquet is placed on the upper arm. A longitudinal incision is made between the fifth and sixth extensor compartment, and muscles are retracted. A 6-hole $2.7-\mathrm{mm}$ plate is contoured and placed on the dorsal surface of the distal ulna (-Fig. 1). After two distal cortical screws are predrilled and inserted, the osteotomy site and the longitudinal axis are marked. The plate is removed, and two parallel transverse cuts are made. The ulna is shortened 4 to $6 \mathrm{~mm}$ to obtain a final ulnar variance of between 0 and $-1 \mathrm{~mm}$. The plate is placed back in position, and the two distal screws are inserted. A screw is placed just proximal from the osteotomy site, and a compression clamp is applied on either site of the osteotomy to achieve further interfragmentary compression. The remaining two screws are placed proximally. Stability of the distal radioulnar joint is tested. If stable, the incision is closed using resorbable sutures.

Postoperatively, patients are immobilized in a forearm cast for 4 weeks, followed by physical hand therapy. After immobilization, no splint was used, and patients could use the wrist and forearm freely.

\section{Statistical Analysis}

We analyzed data using IBM SPSS statistics 21.0. The reliability coefficient, which displays an estimation of the internal consistency of the questionnaire made by the authors, was assessed using Cronbach's $\alpha$. Cronbach's $\alpha$ ranges from zero to one, in which one implies a high internal consistency. Either Pearson's or Spearman's calculations were used to analyze the correlation between variables. Significance was established at a $p$ value of less than 0.05 .

\section{Results}

We identified 34 patients with at least 1-year follow-up. They all underwent USO without an accompanying surgery. 

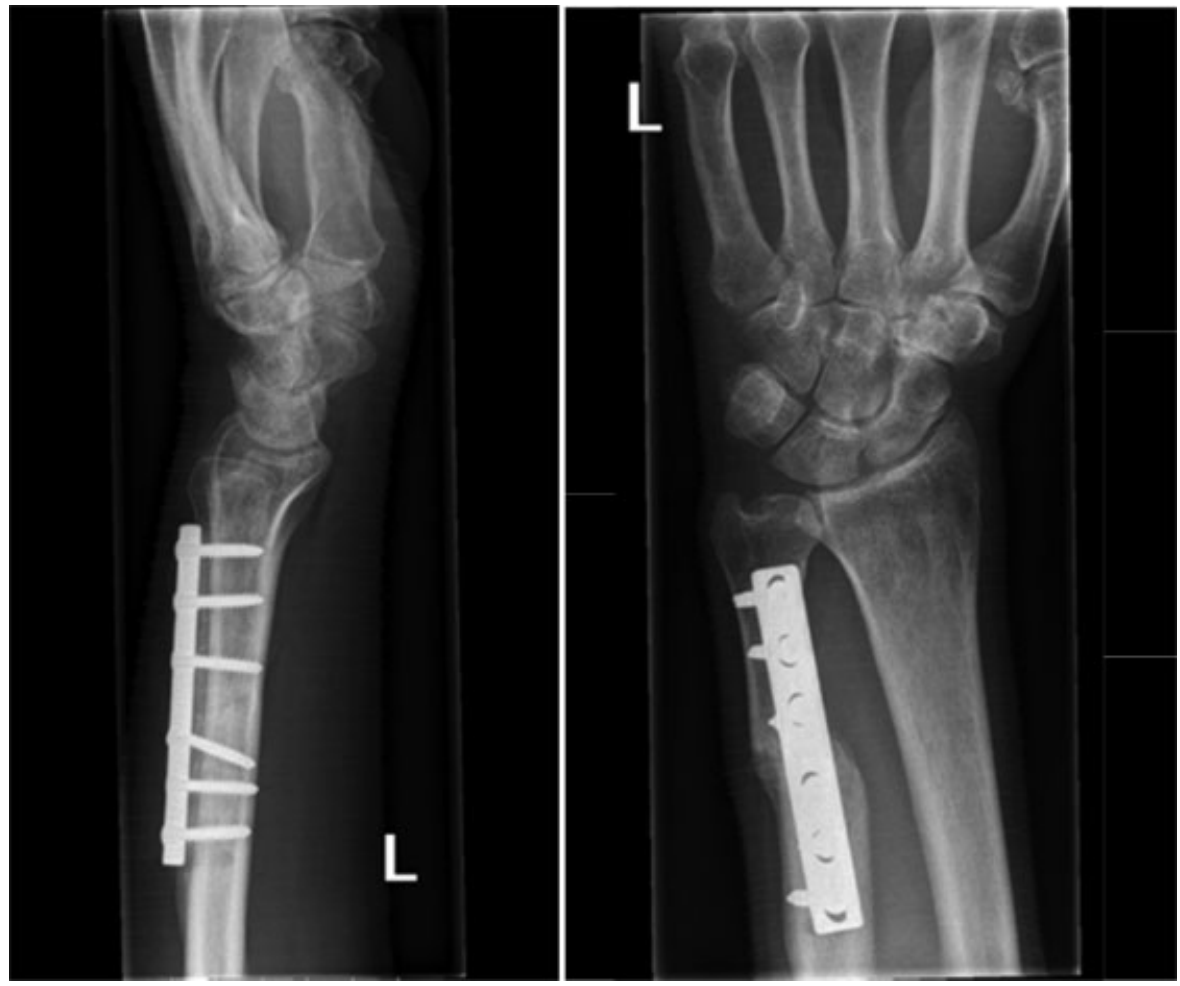

Fig. 1 Lateral and anterior-posterior views after ulnar shortening osteotomy.

All patients had been diagnosed with an ulnar impaction syndrome due to either congenital $(n=19)$ or acquired $(n=15)$ conditions. The symptoms included ulnocarpal tenderness, a painful ulnar stress test and positive ulnar variance on X-ray. Other potential causes of ulnar-sided wrist pain, such as arthrosis or distal radioulnar joint abnormalities had been ruled out (-Fig. 2).

The response rate, of patients enrolled in this study, was $59 \%$ (20/34). The remaining cohort consisted of 6 men and 14 women (total, $n=20$ ); mean age was 43 years (range: $17-$ 72 years, standard deviation [SD]: 15 years). Patients mean age, sex, and side of ulnar shortening is displayed in - Table 1.

The PRWE, DASH, and the patient satisfaction questionnaire were conducted at an average of 3 years and 7 months after USO (range: 10 year-10 months). -Table 2 shows objective and subjective outcomes. The mean postoperative

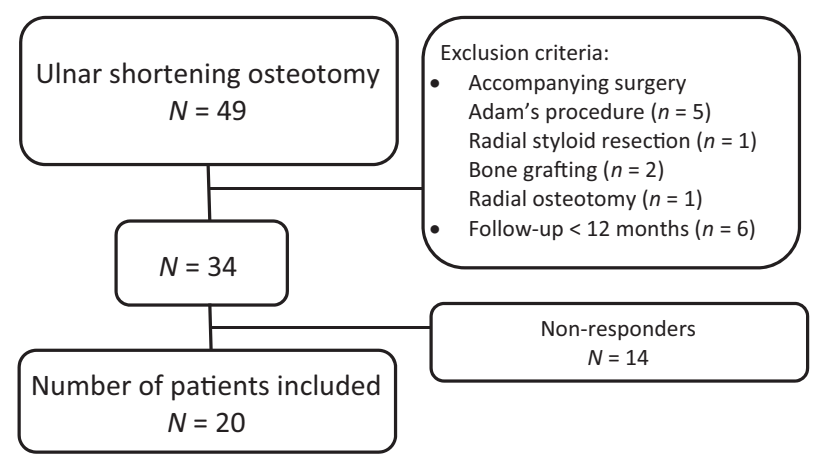

Fig. 2 Flow chart of patient selection.
PRWE score was 28 (SD: 30). Overall, patients were very satisfied with the cosmetic result (mean: 2; SD: 2).

The mean postoperative DASH score was 20 (SD: 26). DASH scores for work were missing in 1 out of 20 (5\%) patients and in 9 out of 20 (45\%) for sports (-Table 2 ).

A total of $75 \%(15 / 20)$ of patients were either very satisfied or satisfied with the operation. Overall, 65\% (13/20) of patients were very satisfied or satisfied with the eventual result of the operation, $80 \%(16 / 20)$ would undergo the same operation again, and $70 \%(14 / 20)$ would recommend the operation.

In one patient (5\%) a complication occurred. In this case, revision surgery with bone grafting was performed after

Table 1 Baseline characteristics

\begin{tabular}{|c|c|c|c|}
\hline & $\begin{array}{l}\text { Male } \\
(n=6)\end{array}$ & $\begin{array}{l}\text { Female } \\
(n=14)\end{array}$ & $\begin{array}{l}\text { Total } \\
(n=20)\end{array}$ \\
\hline $\begin{array}{l}\text { Age at surgery, y } \\
\text { Mean (SD) }\end{array}$ & $40(14)$ & $44(16)$ & $43(15)$ \\
\hline \multicolumn{4}{|l|}{ Operated side, $n$ (\%) } \\
\hline Right & $4(67)$ & $10(71)$ & $14(70)$ \\
\hline Left & $2(33)$ & $4(29)$ & $6(30)$ \\
\hline \multicolumn{4}{|c|}{ Indication for USO, $n$ (\%) } \\
\hline Congenital disorder & $1(17)$ & $10(71)$ & $11(55)$ \\
\hline Acquired & $5(83)$ & $4(29)$ & $9(45)$ \\
\hline
\end{tabular}

Abbreviations: SD, standard deviation; USO, ulnar shortening osteotomy. 
Table 2 Objective and subjective outcomes

\begin{tabular}{|c|c|c|c|}
\hline & $\begin{array}{l}\text { Male } \\
(n=6)\end{array}$ & $\begin{array}{l}\text { Female } \\
(n=14)\end{array}$ & $\begin{array}{l}\text { Total } \\
(n=20)\end{array}$ \\
\hline \multicolumn{4}{|l|}{ DASH score, mean (SD) } \\
\hline Total & $37(35)$ & $13(18)$ & $20(26)$ \\
\hline Work $^{\mathrm{a}}$ & $39(34)$ & $14(22)$ & $22(28)$ \\
\hline Sports $^{\mathrm{b}}$ & 59 (49) & $19(23)$ & $26(31)$ \\
\hline PRWE score, mean (SD) & $45(35)$ & $20(27)$ & $28(31)$ \\
\hline $\begin{array}{l}\text { PRWE cosmetic result, } \\
\text { mean (SD) }\end{array}$ & $3(4)$ & $1(2)$ & $2(2)$ \\
\hline $\begin{array}{l}\text { Removal of hardware, } \\
n(\%)\end{array}$ & $0(0)$ & $6(43)$ & $6(30)$ \\
\hline $\begin{array}{l}\text { Time to removal, mo } \\
\text { Mean (SD) }\end{array}$ & & $25(29)$ & $25(29)$ \\
\hline $\begin{array}{l}\text { Amount of shortening, } \\
\text { mm } \\
\text { Mean (SD) }\end{array}$ & $5.2(0.4)$ & $5.6(0.9)$ & $5.5(0.8)$ \\
\hline \multicolumn{4}{|l|}{ Complications. $n(\%)$} \\
\hline Infection & $0(0)$ & $0(0)$ & $0(0)$ \\
\hline Pseudoarthrosis & $0(0)$ & $0(0)$ & $0(0)$ \\
\hline Plate fracture & $1(17)$ & $0(0)$ & $1(5)$ \\
\hline
\end{tabular}

Abbreviations: DASH, Disability of the Arm, Shoulder, and Hand; PRWE, Patient-Rated Wrist Evaluation; SD, standard deviation.

$a_{n}=13$ women and 19 total.

${ }^{b} n=2$ men, 9 women, and 11 total.

plate breakage as a result of trauma. Union of the osteotomy site occurred in all patients, except one, after 5.3 months (SD: 1.4). The removal of hardware due to irritation was noted in six patients (30\%). Mean postoperative time interval until the removal of hardware was 25 months (SD: 29).

We calculated Cronbach's $\alpha$ for the authors' questionnaire, which was 0.914. Which means the questionnaire has a high internal consistency. Furthermore, a significant correlation was seen between patients satisfaction about the operation and the total DASH and PRWE scores $(\rho=0.481, p=0.032$ and $\rho=0.512, p=0.021$ ).

Also, mean PRWE and DASH scores were calculated for men and women without revision surgery for removal of hardware. Mean DASH and PRWE scores increased for men (DASH: 37-40, PRWE: 45-49) and decreased for women (DASH: 13-8, PRWE: 20-7).

\section{Discussion}

Placement of the hardware in USO can be varied either on the dorsal surface of the ulna, volar surface or ulnar surface. Plate removal, after placement on the volar surface of the ulna, occurs in up to $55 \%$ of patients. ${ }^{5,6,14-18}$ Clark and Geissler, however, described a hardware removal rate of $0 \%$, although $5 \%$ of patients complained about hardware irritation. When plates are placed ulnarly, revision surgery for plate removal is performed in 21 to $32 \%$ of patients. ${ }^{12,15,19,20}$ Loh et al report irritation rates of $68 \%$ placing plates on the ulnar border. ${ }^{21}$ In
Köppel et al requirement of hardware removal was present in $75 \%$ of patients. ${ }^{22}$ The dorsal approach for plate placement is not yet common practice, but results seem promising. Hardware removal rates range from 6 to $25 \%{ }^{6,15,23}$ In our study, the occurrence of hardware removal was $30 \%$.

To evaluate difficulty in performing physical activities, symptoms and psychosocial effects after USO, postoperative DASH scores were evaluated. Scores range from 9 to 37.2 when plates are placed on the volar surface of the ulna. The lowest DASH score is reported at a follow-up of 11 years. ${ }^{14,16,18,24-26}$ After placement of hardware on the ulnar border, postoperative DASH scores range from 21.0 to $37.21 .^{19,27,28}$ No study reported on postoperative DASH scores after application of plates on the dorsal surface. In our study, mean DASH score was 20. Overall DASH scores in our study were measured at a mean follow-up of more than 7 years and lie in the midrange when compared with previously reported scores. Only Fulton et al mentioned DASH scores for work and sports, which are inferior to our DASH scores (mean DASH work: 38 and mean DASH sports: 50 ). ${ }^{19}$

We used the PRWE score to assess pain and evaluate function during normal and special activities after USO. Previously reported PRWE scores vary from 15 to 40.2 for volarly placed plates. ${ }^{6,24,29}$ Only one single study reported on ulnarly placed plates. ${ }^{27}$ Das et al described PRWE scores for dorsally placed plates. In our study, PRWE scores are not better, nor worse than PWRE scores reported in other literature (scores ranging from 15 to 42)..$^{6,24,29}$ Das et al reported no significant difference in PRWE when dorsal plating is compared with volar plating. ${ }^{6}$

When a comparison is made between pre- and postoperative PRWE and DASH scores, a significant reduction of scores is seen. ${ }^{24,27}$ Isaacs et al placed their plates volarly and Kim and Park ulnarly. No results on pre- and postoperative PRWE and DASH scores have been reported on dorsally placed plates.

Over time, many options have been described regarding the osteotomy. It is hypothesized an oblique osteotomy results in the faster bony union and little nonunion cases, due to an increase in surface area. ${ }^{30}$ Many previous studies confirm this hypothesis and describe nonunion rates of 0\%. ${ }^{18,22,24,25,29,31-33}$ However, others report up to $18 \%$ nonunion after performing an oblique osteotomy. $6,14-17,19,21,34-36$ When a transverse osteotomy is performed, nonunion occurs in up to $16 \%$ of patients. $5,12,20,22,29,30,37,38$ In our study a transverse osteotomy was used and only one nonunion occurred. External compression devices may provide more compression and better alignment of the osteotomy, which leads to better and faster union. ${ }^{12,32,35}$ In this study, a free hand technique was used, and union occurred in almost all patients.

There are several limitations to this study. First, a retrospective design was used. No preoperative DASH and PRWE scores were available. Consequently, functional improvement rates could not be assessed. However, patient factors such satisfaction, functional disability, pain and psychosocial effects of the procedure, are useful for estimating long-term outcomes after USO with dorsally placed hardware. Furthermore, the timing of control X-rays was not protocolled, so 
time to the union could not be assessed. However, only one nonunion occurred. Another limitation is the possible occurrence of nonresponse bias, due to the response rate of $59 \%$. Only two minor complications occurred among nonresponders. Finally, the sample size of this study is small, which reduces the chance of detecting true effects. To evaluate real differences between hardware irritation due to the localization of plate placement, prospective studies with larger patient populations are needed.

In conclusion, no differences are seen in complication rates, PRWE and DASH scores. The incidence of hardware removal, in patients undergoing a USO with a dorsally placed plate for ulnar impaction syndrome, lies in the midrange when compared with previously described results for dorsally, ulnarly, and volarly placed plates. Overall, patients were satisfied with the operation and the results. Based on the results of our study, we would recommend considering a freehand technique with transverse osteotomy and plate placement on the dorsal surface of the ulna as an option for ulnar shortening, for this is a relatively easy method, patients' outcomes are comparable to other techniques, and patients are satisfied.

Note

The authors obtained approval of the medical ethical review committee.

Funding

None.

Conflict of Interest

None.

\section{Acknowledgment}

The authors thank Peter Zuidhoff, PhD, Department of Biostatistics and Research Support, University Medical Centre, Utrecht for advice on statistics.

\section{References}

1 Nagy L, Jungwirth-Weinberger A, Campbell D, Pino JG. The AO ulnar shortening osteotomy system indications and surgical technique. J Wrist Surg 2014;3(02):91-97

2 Fricker R, Pfeiffer KM, Troeger H. Ulnar shortening osteotomy in posttraumatic ulnar impaction syndrome. Arch Orthop Trauma Surg 1996;115(3-4):158-161

3 Sachar K. Ulnar-sided wrist pain: evaluation and treatment of triangular fibrocartilage complex tears, ulnocarpal impaction syndrome, and lunotriquetral ligament tears. J Hand Surg Am 2012;37(07):1489-1500

4 Aminian A, Schoenecker PL. Premature closure of the distal radial physis after fracture of the distal radial metaphysis. J Pediatr Orthop 1995;15(04):495-498

5 Iwasaki N, Ishikawa J, Kato H, Minami M, Minami A. Factors affecting results of ulnar shortening for ulnar impaction syndrome. Clin Orthop Relat Res 2007;465(465):215-219

6 Das De S, Johnsen PH, Wolfe SW. Soft tissue complications of dorsal versus volar plating for ulnar shortening osteotomy. J Hand Surg Am 2015;40(05):928-933

7 Stockton DJ, Pelletier ME, Pike JM. Operative treatment of ulnar impaction syndrome: a systematic review. J Hand Surg Eur Vol 2015;40(05):470-476
8 Chun S, Palmer AK. The ulnar impaction syndrome: follow-up of ulnar shortening osteotomy. J Hand Surg Am 1993;18(01):46-53

9 Changulani M, Okonkwo U, Keswani T, Kalairajah Y. Outcome evaluation measures for wrist and hand: which one to choose? Int Orthop 2008;32(01):1-6

10 Brink SM, Voskamp EG, Houpt P, Emmelot CH. Psychometric properties of the Patient Rated Wrist/Hand Evaluation-Dutch Language Version (PRWH/E-DLV). J Hand Surg Eur Vol 2009;34 (04):556-557

11 Schuurman AH, Sleegers E. The quick DASH outcome measure DLV (2003). Available at: http://www.dash.iwh.on.ca/sites/dash/public/translations/QuickDASH_Dutch.pdf. Accessed September 29, 2014

12 Wehbé MA, Cautilli DA. Ulnar shortening using the AO small distractor. J Hand Surg Am 1995;20(06):959-964

13 Milch H. Cuff resection of the ulna for malunited Colles' fracture. J Bone Joint Surg 1941;39:311-313

14 Doherty C, Gan BS, Grewal R. Ulnar shortening osteotomy for ulnar impaction syndrome. J Wrist Surg 2014;3(02):85-90

15 Fufa DT, Carlson MG, Calfee RP, Sriram N, Gelberman RH, Weiland AJ. Mid-term results following ulna shortening osteotomy. HSS J 2014;10(01):13-17

16 Srinivasan RC, Jain D, Richard MJ, Leversedge FJ, Mithani SK, Ruch DS. Isolated ulnar shortening osteotomy for the treatment of extra-articular distal radius malunion. J Hand Surg Am 2013;38 (06):1106-1110

17 Ahsan ZS, Song Y, Yao J. Outcomes of ulnar shortening osteotomy fixed with a dynamic compression system. J Hand Surg Am 2013; 38(08):1520-1523

18 Luria S, Lauder AJ, Trumble TE. Comparison of ulnar-shortening osteotomy with a new Trimed dynamic compression system versus the Synthes dynamic compression system: clinical study. J Hand Surg Am 2008;33(09):1493-1497

19 Fulton C, Grewal R, Faber KJ, Roth J, Gan BS. Outcome analysis of ulnar shortening osteotomy for ulnar impaction syndrome. Can J Plast Surg 2012;20(01):e1-e5

20 Lautenbach M, Millrose M, Schmidt NS, Zach A, Eichenauer F, Eisenschenk A. Ulnocarpal impaction syndrome: treatment with a transverse ulnar shortening osteotomy from an ulnodorsal approach. Arch Orthop Trauma Surg 2014;134(06):881-885

21 Loh YC, Van Den Abbeele K, Stanley JK, Trail IA. The results of ulnar shortening for ulnar impaction syndrome. J Hand Surg $[\mathrm{Br}] 1999$; 24(03):316-320

22 Köppel M, Hargreaves IC, Herbert TJ. Ulnar shortening osteotomy for ulnar carpal instability and ulnar carpal impaction. J Hand Surg Am 1997;22B(04):451-456

23 Huang HK, Wang JP, Wang ST, Huang YC, Liu CL. Freehand technique with the predrilled hole method for ulnar-shortening osteotomy. J Chin Med Assoc 2016;79(02):77-82

24 Isaacs J, Howard SB, Gulkin D. A prospective study on the initial results of a low profile ulna shortening osteotomy system. Hand (NY) 2010;5(02):148-154

25 Kitzinger HB, Karle B, Löw S, Krimmer H. Ulnar shortening osteotomy with a premounted sliding-hole plate. Ann Plast Surg 2007;58(06):636-639

26 Tatebe M, Shinohara T, Okui N, Yamamoto M, Hirata H, Imaeda T. Clinical, radiographic, and arthroscopic outcomes after ulnar shortening osteotomy: a long-term follow-up study. J Hand Surg Am 2012;37(12):2468-2474

27 Kim JK, Park ES. Comparative responsiveness and minimal clinically important differences for idiopathic ulnar impaction syndrome. Clin Orthop Relat Res 2013;471(05):1406-1411

28 Moermans A, Degreef I, De Smet L. Ulnar shortening osteotomy for ulnar ideopathic impaction syndrome. Scand J Plast Reconstr Surg Hand Surg 2007;41(06):310-314

29 Rajgopal R, Roth J, King G, Faber K, Grewal R. Outcomes and complications of ulnar shortening osteotomy: an institutional review. Hand (NY) 2015;10(03):535-540 
30 Rayhack JM, Gasser SI, Latta LL, Ouellette EA, Milne EL. Precision oblique osteotomy for shortening of the ulna. J Hand Surg Am 1993;18(05):908-918

31 Clark SM, Geissler WB. Results of ulnar shortening osteotomy with a new plate compression system. Hand (NY) 2012;7(03):281-285

32 Chen NC, Wolfe SW. Ulna shortening osteotomy using a compression device. J Hand Surg Am 2003;28(01):88-93

33 Renfree KJ, Odgers RA. Stacked-blade, single-cut, ulnar-shortening osteotomy. Orthopedics 2015;38(02):e80-e87

34 Tränkle M, van Schoonhoven J, Krimmer H, Lanz U. Indication and results of ulna shortening osteotomy in ulnocarpal wrist joint pain [in German]. Unfallchirurg 2000;103(03):197-202
35 Pouliot M, Yao J. Ulnar shortening osteotomy utilizing a TriMed ulnar osteotomy system. Tech Hand Up Extrem Surg 2014;18(02): $72-76$

36 Schmidle G, Arora R, Gabl M. Ulnar shortening with the ulna osteotomy locking plate. Oper Orthop Traumatol 2012;24(03): 284-292

37 Hamada Y, Sairyo K, Hibino N, Kobayashi A, Sato R. Effect of preservation of corticoperiosteal attachment on bone healing at osteotomy sites after ulna-shortening osteotomy. Hand (NY) 2015;10(01):105-110

38 Nagle DJ, Bernstein MA. Laser-assisted arthroscopic ulnar shortening. Arthroscopy 2002;18(09):1046-1051 


\section{Appendix}

Questionnaire satisfaction

In the past you underwent an ulnar shortening osteotomy in the University Medical Centre Utrecht. The questions below are about this operation. Choose the most applicable answer. Please answer all questions.

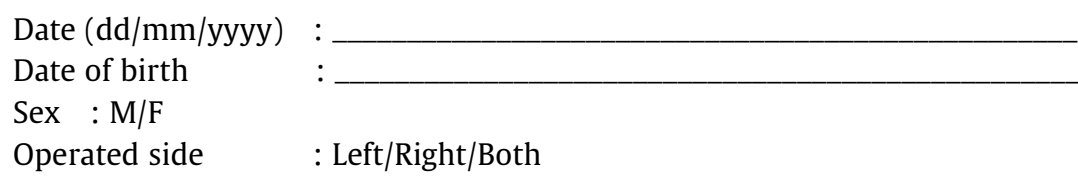

1. I am satisfied about the operation performed.

I totally agree I agree I don't agree/disagree I disagree I totally disagree

2. If necessary, I would choose the same operation again.

I totally agree I agree I don't agree/disagree I disagree I totally disagree

3. I would recommend this surgery.

I totally agree I agree I don't agree/disagree I disagree I totally disagree

4. I am satisfied about the final result of the operation

I totally agree I agree I don't agree/disagree I disagree I totally disagree

Comments: 